\title{
Magnetohydrodynamic Waves and Seismology of the Solar Atmosphere
}

\author{
Robertus Erdélyi • Marcel Goossens
}

Published online: 17 June 2011

(C) Springer Science+Business Media B.V. 2011

The solar atmosphere is a gigantic plasma physics laboratory that has attracted attention from plasma physicists for over a century. It extends from the visible surface of the Sun or photosphere, into the corona and solar wind all the way to the Earth and beyond. The plasma of the solar atmosphere has properties that makes it a real challenge to study and fully understand. It is structured, inhomogeneous, highly dynamic and is characterized by a large range of spatial and temporal scales. Among the many unsolved questions of modern solar plasma physics a few emerge with importance well beyond the boundaries of solar physics. How is plasma in the corona heated to multi-million $\mathrm{K}$ temperatures? What are the fundamental underlying physical processes of plasma acceleration and solar wind generation? What are the physical processes that drive Space Weather?

It has been predicted for several decades that magnetohydrodynamic (MHD) waves may play a key role in processes that determine the physical conditions in the solar atmosphere. However, due to the lack of adequate instrumental resolution there was only limited or indirect evidence for the existence of MHD waves. The actual discovery of magnetic or magnetically dominated waves with imaging and spectro-polarimetric instrumentation onboard of solar satellites supplemented with high-resolution ground-based instrumentation since the mid-1990s and into the new milenium has shed new light on MHD waves. Slow and fast magneto-acoustic waves and Alfvén waves, predicted by theory, have now been observed in a wide variety of magnetic structures in the solar atmosphere. Research on MHD waves has evolved over the last two decades from a largely theoretical enterprise with little observational underpinning into a subject that is now driven by a wealth of observations which show that MHD waves are almost everywhere in the solar atmosphere. New theoretical developments are needed to understand and exploit the available observations. MHD waves

R. Erdélyi (凶)

Solar Physics and Space Plasma Research Centre (SP2RC), The University of Sheffield, Hicks

Building, Hounsfield Road, S3 7RH, Sheffield, England, UK

e-mail: robertus@sheffield.ac.uk

M. Goossens

Centre voor Plasma-Astrophysica, Katholieke Universiteit Leuven, Leuven, 3001, Belgium 
are important for various reasons. They reflect the stable dynamic behaviour of the plasma structures they live in. They transport energy and can heat plasma. Equally importantly, they provide us with an indirect technique for probing the plasma in which they occur. With the accurate determination of basic properties of MHD waves from observations, such as periods, damping times and amplitudes, came the natural realization that MHD waves could be used in the same way as seismic waves are used to probe the interior of the Earth, to probe the plasma of the solar atmosphere. This indirect technique is known as seismology and is used for the interior of the Earth, the interior of the sun (helio-seismology) and the interior of stars (stellar seismology). Seismology of the solar atmosphere makes it possible to determine properties of the magnetized solar plasma that cannot be directly measured with great accuracy, examples are the strength of the magnetic field, the scale heights of density and magnetic field. The hope is that seismology will help us to understand the nature of heating processes, the structuring of the magnetic field, plasma temperature, shape and extent of flux tube or active regions, etc.

Solar magneto-seismology is based on comparing observed with theoretical values of particular wave variables. It requires accurate observations and sufficiently advanced theoretical MHD wave models. The latest high spatial/temporal resolution wave observations from the new generation of space-borne and ground-based instruments, e.g., Hinode, Solar Dynamics Observatory (SDO) and Rapid Oscillations in the Solar Atmosphere (ROSA) are a tremendous impetus for advancing our study of MHD waves and for adding more physical complexity to the theoretical MHD wave models. The aim and hope is that new theoretical developments, both by analytical and numerical modelling, will help pave the way for the interpretation of data from future instruments, e.g., Advanced Technology Solar Telescope (ATST), Solar Orbiter (SO) or HiRISE.

The timeliness of research on MHD waves in the solar atmosphere cannot be overstated, with the international race now on to explain the wide variety of Alfvén and magnetoacoustic waves observed recently with the Coronal Multi-channel Polarimeter (CoMP) and Hinode in the lower and upper solar atmosphere. This Volume, in the format of a Topical Issue, aims to summarize the status of our current understanding of MHD waves in the solar atmosphere. It presents the current trends, achievements and modern challenges written in twelve specialised review papers by experts in the field. This topical volume is largely based on the presentations and discussions that took place at BUKS2009, 6-8 April 2009, Leuven, Belgium, a "Workshop on MHD Waves and Seismology of the Solar Atmosphere". The authors and guest editors are grateful to BUKS2009 for the support and for an inspiring and stimulating working environment. The members of the LOC and SOC played a pivotal role in this respect.

It is a pleasure to acknowledge financial support for BUKS 2009 by FWO-Vlaanderen, the Research Council of the K.U.Leuven and the Faculty of Sciences of the K.U.Leuven. Special gratitude goes to the Department of Mathematics of the K.U.Leuven for its secretarial and organisational support that helped create a very enjoyable workshop. In particular it is a pleasure to thank the secretarial staff of the Department of Mathematics.

Guest Editors

Robertus Erdélyi and Marcel Goossens 